\title{
The Canonical Luminous Blue Variable AG Car and Its Neighbor Hen 3-519 Are Much Closer than Previously Assumed
}

\author{
Nathan Smith ${ }^{1}$ and Keivan G. Stassun ${ }^{2,3}$ \\ ${ }^{1}$ Steward Observatory, University of Arizona, 933 North Cherry Avenue, Tucson, AZ 85721, USA \\ ${ }^{2}$ Vanderbilt University, Department of Physics \& Astronomy, 6301 Stevenson Center Lane, Nashville, TN 37235, USA \\ ${ }^{3}$ Fisk University, Department of Physics, 1000 17th Avenue N., Nashville, TN 37208, USA \\ Received 2016 October 20; revised 2017 January 15; accepted 2017 January 27; published 2017 February 24
}

\begin{abstract}
The strong mass loss of Luminous Blue Variables (LBVs) is thought to play a critical role in massive-star evolution, but their place in the evolutionary sequence remains debated. A key to understanding their peculiar instability is their high observed luminosities, which often depends on uncertain distances. Here we report direct distances and space motions of four canonical Milky Way LBVs-AG Car, HR Car, HD 168607, and (candidate) Hen 3-519-from the Gaia first data release. Whereas the distances of HR Car and HD 168607 are consistent with previous literature estimates within the considerable uncertainties, Hen 3-519 and AG Car, both at $\sim 2 \mathrm{kpc}$, are much closer than the 6-8 kpc distances previously assumed. As a result, Hen 3-519 moves far from the locus of LBVs on the Hertzsprung-Russell diagram, making it a much less luminous object. For AG Car, considered a defining example of a classical LBV, its lower luminosity would also move it off the S Dor instability strip. Lower luminosities allow both AG Car and Hen 3-519 to have passed through a previous red supergiant phase, lower the mass estimates for their shell nebulae, and imply that binary evolution is needed to account for their peculiarities. These results may also impact our understanding of LBVs as potential supernova progenitors and their isolated environments. Improved distances will be provided in the Gaia second data release, which will include additional LBVs. AG Car and Hen 3-519 hint that this new information may alter our traditional view of LBVs.
\end{abstract}

Key words: stars: variables: S Doradus

\section{Introduction}

Eruptive mass loss exhibited by luminous blue variables (LBVs) is thought to be important for the evolution of massive stars (Humphreys \& Davidson 1994; Smith \& Owocki 2006; Smith 2014), but the exact role LBVs play and the physics of their instability has been challenging to understand. The class of LBVs defined in the Milky Way and Magellanic Clouds (LMC/SMC) is thought to be responsible for some extragalactic non-supernova (SN) transients (Smith et al. 2011; Van Dyk \& Matheson 2012). The episodic mass loss of LBVs has also been a reference point for interpreting the dense circumstellar material (CSM) around Type IIn supernovae (SNe IIn), which is thought to be indicative of extreme pre-SN eruptions (Smith 2014).

The diverse collection of objects known collectively as LBVs was first proposed as a group by Conti (1984), and the now standard interpretation of these stars and their role in evolution was established through the 1980s and 1990s. The review by Humphreys \& Davidson (1994) provides a summary of the traditional view of LBVs, which has a few key tenets:

1. LBVs are thought to represent a brief transitional phase in the evolution of the most massive stars, between the main sequence O-type stars and the H-deficient Wolf-Rayet (WR) stars. Because they have already lost some mass and because their core luminosity has increased, their $L / M$ ratio is high. This proximity to the classical Eddington limit, combined with Fe opacity, leads to a violent instability in the envelope that somehow triggers runaway Geyser-like mass loss. The heavy mass loss of LBVs, in the single-star view, is essential to remove the $\mathrm{H}$ envelope to form WR stars.

2. Observed S Doradus-type variations, as exemplified most clearly by AG Car in the Milky Way and R127 in the LMC
(Wolf \& Stahl 1982; Stahl et al. 1983), are temperature variations at constant bolometric luminosity. The cool (visibly bright) state is caused by a pseudo-photosphere that develops in an optically thick wind, which occurs at the same temperature regardless of luminosity (Davidson 1987; Humphreys \& Davidson 1994). In their quiescent (hot) phase, all LBVs reside on the diagonal "S Doradus instability strip" (Wolf 1989) on the Hertzsprung-Russell (HR) diagram.

3. The strong mass loss of LBVs halts their redward evolution, preventing them from becoming red supergiants (RSGs). This explains the observed absence of high-luminosity RSGs above $\log \left(L / L_{\odot}\right)=5.8$, due to instability and mass loss in evolved massive single stars.

4. Some LBVs suffer dramatic "giant eruptions" where huge amounts of mass can be lost. Although there is a formalism to explain the mechanism of this mass loss with super-Eddington continuum-driven winds (Owocki et al. 2004), the underlying reason why single stars suddenly exceed the classical Eddington limit by factors of 5-10 has remained unexplained. Nevertheless, this tremendous mass loss is observed and is likely to be important in the evolution of massive stars.

Several aspects of this traditional view, however, have unraveled with time. In particular, the important conjecture that $S$ Dor variations are major mass-loss events caused by optically thick winds appears to be wrong, and steady superEddington winds might not be the dominant driving mechanism in many giant LBV eruptions (see review by Smith 2014). Moreover, the recognition that SNe IIn have progenitors consistent with LBVs (see, e.g., Mauerhan et al. 2013) casts doubt on the idea that LBVs are only a brief transitional phase before the start of core He burning. Investigations with quantitative spectroscopy (de Koter et al. 1996; Groh et al. 2009a, 2009b) disproved the conjecture that 
S Dor brightening events are caused by pseudo-photospheres in optically thick winds (Davidson 1987). The mass-loss rates of $\mathrm{S}$ Dor maxima are not high enough to make such extreme pseudo-photospheres, and so they are more likely to be caused by envelope inflation or pulsation (Graefener et al. 2012). Also, bolometric luminosities during $\mathrm{S}$ Dor eruptions are not necessarily constant (Groh et al. 2009a). Similarly, the idea that giant-eruption maxima are caused by pseudo-photospheres in super-Eddington winds is challenged by light-echo spectra of $\eta$ Carinae (Rest et al. 2012; Prieto et al. 2014; although see Owocki \& Shaviv 2016), by detailed analysis of the ejecta around $\eta$ Car that are better matched by an explosive event (Smith 2006b, 2008, 2013), and the fact that many extragalactic giant LBV eruptions are relatively hot, rather than cool, at peak luminosity (Smith et al. 2011; Mauerhan et al. 2015). Last, as discussed by Smith \& Tombleson (2015), their isolation from massive O-type stars suggests that they are much older than expected. Smith \& Tombleson (2015) suggested that they are largely products of binary evolution and not a transitional state in the lives of the most massive single stars.

Here we add another possible wrinkle to the unraveling story of LBVs. One of the canonical, classical high-luminosity LBVs in the Milky Way is AG Carinae; from a direct measurement of its parallax from Gaia this star appears to be much closer-and therefore much less luminous - than previously thought. Even though AG Car is a defining S Doradus variable, this closer distance moves it well below the $\mathrm{S}$ Doradus instability strip established for LBVs in the LMC (Wolf 1989). Although the distance to LBVs in the LMC is not that uncertain, the distance to Galactic LBVs has always been precarious. Below we discuss AG Car and the three other Galactic LBV-like stars that are included in the Gaia first data release.

The Gaia first data release (DR1) has recently provided trigonometric parallaxes and proper motions for the $\sim 2$ million stars previously observed by Hipparcos and Tycho-2, with a typical precision in the parallax of $\sim 0.25$ mas (Gaia Collaboration et al. 2016). These parallaxes are enabling a number of investigations not previously possible, such as direct measurement of the basic properties of all planets and their stellar hosts (Stassun et al. 2016). These fundamental new measurements also provide an opportunity to reassess the nature of these LBVs.

The data from Gaia DR1 and from the literature that we use in this study are described in Section 2, which in particular includes parallaxes and proper motions for the four Milky Way LBVs Hen 3-519, HD 168607, HR Car, and AG Car. Section 3 presents the principal results of this study, namely, the distances, space motions, and other basic characteristics of these LBVs, as well as the uncertainty in these values. Section 4 discusses the implications of our findings, especially the distances for AG Car and Hen 3-519, which we find to be much closer than previously thought. We conclude with a brief summary of our results in Section 5.

\section{Data and Methods}

We searched the Gaia DR1 catalog for all of the Milky Way LBVs and LBV candidates listed in Smith \& Tombleson (2015), which yielded parallaxes and proper motions for four: Hen 3-519, HD 168607, HR Car, and AG Car (see Table 1). From the parallax, $\pi$, a distance may be straightforwardly computed via $d=1 / \pi$. The parallaxes of the four LBVs span the range $0.34-1.02$ mas, corresponding to distances spanning the range $\approx 1-3 \mathrm{kpc}$.

However, because the parallax measurement errors are relatively large $(28 \%-72 \%)$, the distances and their uncertainties are expected to depend on the adopted prior (see, e.g., Bailer-Jones 2015). Therefore, we have also retrieved the distances computed by Astraatmadja \& Bailer-Jones (2016) using a more realistic prior distribution based on simple but empirically motivated stellar density distributions for the Milky Way. These distances, which span the range $1.2-2.3 \mathrm{kpc}$ (Table 1), are consistent within the uncertainties with those obtained simply via $1 / \pi$, but with somewhat smaller uncertainties due to the more informative prior used. Thus we prefer the Astraatmadja \& Bailer-Jones (2016, hereafter, $\mathrm{ABJ})$ distances in what follows but note that our primary conclusions do not depend strongly on this choice.

The Gaia DR1 release notes state that the parallaxes may possess uncharacterized systematic uncertainties of up to 0.3 mas, and recommend adding an additional 0.3 mas to the reported measurement uncertainty. Consequently, ABJ provide a version of their catalog that includes this additional 0.3 mas error. However, Stassun \& Torres (2016b) used a set of nearby, benchmark eclipsing binary stars (Stassun \& Torres 2016a) to quantify the systematic error, finding it to be $-0.25 \pm 0.05$ mas in the sense that the Gaia parallaxes are systematically too small (distances too long). Jao et al. (2016) found a similar offset among a sample of nearby, high-proper-motion stars in the solar neighborhood. However, at larger distances, Stassun \& Torres (2016a) found that the systematic offset vanishes for $\pi \lesssim 1$ mas $(d \gtrsim 1 \mathrm{kpc})$. This is corroborated by Casertano et al. (2016), who found excellent agreement between the Gaia distances and a large sample of Galactic Cepheids at $d \sim 2 \mathrm{kpc}$. Lindegren et al. (2016) and Sesar et al. (2016) similarly use samples such as RR Lyrae stars at intermediate distances to argue that no correction is needed beyond $\sim 1 \mathrm{kpc}$. Finally, Davies et al. (2017) uses red clump stars over a large range of galactic distances to broadly confirm the above findings, with an offset similar to that of Stassun \& Torres (2016a) for nearby stars that vanishes at $d \gtrsim 1.2 \mathrm{kpc}$. Therefore, since the distances of the LBVs in our study sample are all greater than $\sim 1 \mathrm{kpc}$ (Table 1), we do not apply any offset to the parallaxes and moreover do not add any further systematic error to the reported measurement uncertainties.

\section{Results}

$H D$ 168607: This LBV is located only about $1^{\prime}$ away from HD 168625, which is well-known for its SN 1987A-like triplering nebula (Smith 2007). HD 168607 is considered an LBV based on its characteristic variability, whereas its neighbor HD 168625 is usually considered an LBV candidate (Sterken et al. 1999). Both are found in the outskirts of the star-forming region M17 and are thought to be part of the larger Ser OB1 association at $\sim 2.2 \mathrm{kpc}$ (Chentsov \& Gorda 2004). A distance of $\sim 2.2 \mathrm{kpc}$ is usually adopted in the literature (van Genderen et al. 1992), although distances of $1.2-2.8 \mathrm{kpc}$ have been proposed (Robberto \& Herbst 1998; Pasquali et al. 2002). The Gaia DR1 distance to HD 168607 from Table 1 is $0.98 \pm 0.27 \mathrm{kpc}(\pi=1.02 \pm 0.28 \mathrm{mas})$ or $1.16 \pm 0.35 \mathrm{kpc}$ ( $\pi=0.87 \pm 0.26$ mas) using the ABJ parallaxes. This is on the low end of previously adopted values that are usually close to $2 \mathrm{kpc}$, but not too far off. The $95 \%$ upper limit from the ABJ parallaxes is $1.9 \mathrm{kpc}$. This is unlikely to prompt a major 
Table 1

Data Used for the LBVs in Our Study Sample

\begin{tabular}{|c|c|c|c|c|c|c|c|c|c|c|c|c|}
\hline \multirow{2}{*}{ Name } & \multirow{2}{*}{ Hipp. ID } & \multirow{2}{*}{ TYC ID } & \multicolumn{6}{|c|}{ Gaia DR1 } & \multicolumn{4}{|c|}{ Astraatmadja \& Bailer-Jones (2016) } \\
\hline & & & $\pi$ (mas) & $\sigma_{\pi}$ (mas) & $\begin{array}{c}\mu_{\alpha} \\
\left(\operatorname{mas} \mathrm{yr}^{-1}\right)\end{array}$ & $\begin{array}{l}(\operatorname{mas} \\
\left.\mathrm{yr}^{-1}\right)\end{array}$ & $\begin{array}{c}\mu_{\delta} \\
\left(\operatorname{mas}^{-1} r^{-1}\right)\end{array}$ & $\begin{array}{l}(\operatorname{mas} \\
\left.\mathrm{yr}^{-1}\right)\end{array}$ & $d(\mathrm{pc})$ & $\sigma_{d}(\mathrm{pc})$ & $d_{5 \%}(\mathrm{pc})$ & $d_{95 \%}(\mathrm{pc}$ \\
\hline Hen 3-519 & $\ldots$ & $\begin{array}{c}8958- \\
1166-1\end{array}$ & 0.796 & 0.575 & -4.282 & 1.799 & 3.904 & 0.853 & 1600 & 859 & 750 & 3575 \\
\hline HD 168607 & 89956 & $\ldots$ & 1.016 & 0.282 & 0.404 & 0.102 & -1.314 & 0.063 & 1156 & 346 & 754 & 1891 \\
\hline
\end{tabular}

revision in the interpretation of this object. We postpone a detailed discussion until the higher precision that will be available in the next Gaia data release. HD 168607 appears to have a low transverse velocity of only a few $\mathrm{km} \mathrm{s}^{-1}$ indicated by its Gaia proper motion.

HR Car: The distance adopted in the literature for HR Car is usually $5 \pm 1 \mathrm{kpc}$ (e.g., van Genderen et al. 1991; Groh et al. 2009a), placing it among the low-luminosity group of LBVs (see, e.g., Smith et al. 2004). The Gaia distance in Table 1 is $2.92 \pm 2.04 \mathrm{kpc}(\pi=0.34 \pm 0.24$ mas $)$ or $2.27 \pm 0.97 \mathrm{kpc}$ ( $\pi=0.44 \pm 0.19$ mas) according to the ABJ parallaxes, which is lower than previously assumed, but the $95 \%$ upper limit of $4.6 \mathrm{kpc}$ is arguably consistent with the usual estimate. Given that HR Car is also now known to have a resolved wide companion about 3 mas away (Boffin et al. 2016), which may complicate the measured parallax, we do not advocate a major revision of its distance or luminosity at this time. Again, we postpone a detailed discussion pending the higher precision that will be available in the next Gaia data release. The Gaia absolute proper motion of HR Car is about $6.5 \pm 0.16 \mathrm{mas} \mathrm{yr}^{-1}$ $\left(\mathrm{PA} \approx 297^{\circ}\right.$ ), which, at a distance of $\sim 2.5 \mathrm{kpc}$, translates to a transverse velocity of about $72 \mathrm{~km} \mathrm{~s}^{-1}$.

$A G$ Car: Although AG Car is seen in projection amid the Car OB1/OB2 association, located at $2-2.5 \mathrm{kpc}$, a larger distance has usually been adopted in the literature, making AG Car one of the most luminous stars in the Milky Way. The larger distance is based on its radial velocity relative to the local standard of rest (LSR) compared to the Galactic rotation curve, as well as its high line-of-sight extinction, from which Humphreys et al. (1989) derived a likely distance of $6.4-6.9 \mathrm{kpc}$. However, the new Gaia distance is $2.50 \pm$ $1.41 \mathrm{kpc}(\pi=0.40 \pm 0.23 \mathrm{mas})$ or $1.95 \pm 0.73 \mathrm{kpc}(\pi=0.51$ \pm 0.19 mas) according to the ABJ parallaxes, with a $95 \%$ upper limit of $3.7 \mathrm{kpc}$ (Table 1). This would appear to rule out the larger distance above $6 \mathrm{kpc}$ derived by Humphreys et al. (1989), instead suggesting membership in the closer Car OB1/ OB2 association after all. If even approximately correct, this much closer distance has profound implications for our interpretation of $\mathrm{AG}$ Car, and consequences for our understanding of LBVs in general, as discussed in the next section. At a distance of $\sim 2 \mathrm{kpc}$, the measured Gaia absolute proper motion of $5.2 \pm 0.26 \mathrm{mas} \mathrm{yr}^{-1}$ (to the west/northwest; $\mathrm{PA} \approx 292^{\circ}$ ) would suggest a transverse velocity of about $50 \mathrm{~km} \mathrm{~s}^{-1}$.

Hen 3-519: Hen 3-519 is located very close in the sky to AG Car (about 20' away) and would appear to be part of the same Car OB1/OB2 association, but like AG Car, previous authors have generally favored a very large distance near $8 \mathrm{kpc}$. The large $8 \mathrm{kpc}$ distance was proposed by Davidson et al.
(1993) based on the large line-of-sight extinction inferred from UV data. Smith et al. (1994) found a slightly lower reddening, but also favored a large distance near $8 \mathrm{kpc}$ based on the LSR velocity implied by nebular emission and interstellar absorption lines in high-resolution spectra. Again, this would make Hen 3519 an extremely luminous star. Like AG Car, however, parallax once again indicates a much smaller distance. The Gaia distance is $1.26 \pm 0.91 \mathrm{kpc}(\pi=0.80 \pm 0.58 \mathrm{mas})$ or $1.60 \pm 0.86 \mathrm{kpc}$ ( $\pi=0.63 \pm 0.34$ mas $)$ according to the $\mathrm{ABJ}$ parallaxes, with a $95 \%$ upper limit from the ABJ parallaxes of $3.6 \mathrm{kpc}$ (Table 1). This would appear to rule out the larger distance of $8 \mathrm{kpc}$ proposed by Davidson et al. (1993), instead suggesting membership in the closer $\mathrm{Car}$ OB1/OB2 association, just like its neighbor $\mathrm{AG}$ Car. At a distance of $\sim 2 \mathrm{kpc}$, the measured Gaia absolute proper motion of $5.8 \pm 2.7 \mathrm{mas} \mathrm{yr}^{-1}$ $\left(\mathrm{PA} \approx 312^{\circ}\right.$ ) would suggest a transverse velocity of $56 \mathrm{~km} \mathrm{~s}^{-1}$. This is, again, very similar in magnitude and direction to the motion of AG Car, although with larger uncertainty.

The three targets in Carina all exhibit a similar absolute proper motion of roughly $5 \mathrm{mas} \mathrm{yr}^{-1}$ to the west/northwest. This corresponds to apparent motion in a direction along the Galactic plane. It is roughly consistent with the direction and magnitude of longitude drift expected for Galactic rotation on circular orbits in the solar neighborhood. At $\sim 2 \mathrm{kpc}$, objects on the near side of the Carina Arm are at roughly the same radius from the Galactic Center as the Sun. At a much larger distance of 6-8 kpc and a location on the far side of the Carina Arm, the expected proper motions should be less, so perhaps the improved precision of future Gaia data releases will provide a more definitive constraint from proper motion.

As a further check on the veracity of the Gaia distances for these stars, we used the sample of $\mathrm{O}$ stars in the Carina starforming region ${ }^{4}$ from Smith (2006a), which are all expected to be at a common distance of $\sim 2.2 \mathrm{kpc}(\pi \sim 0.45 \mathrm{mas})$ on the basis of multiple lines of evidence, including the nebular expansion of $\eta$ Car itself (see, e.g., Smith 2006b). Forty-three of the stars in that study are present in the Gaia DR1, and in Figure 1 we present the distribution of their parallaxes using both the direct Gaia DR1 parallaxes and the Milky Way priorbased parallaxes from ABJ. The former gives a mean distance of $2.06 \pm 0.41 \mathrm{kpc}$ and the latter gives $1.86 \pm 0.05 \mathrm{kpc}$. The

\footnotetext{
4 Note that we are not making this comparison to suggest that AG Car or Hen 3-519 are necessarily members of the Carina OB association (although they might be), and we do not depend upon this assumption. Instead, we are using the Carina Nebula O-type stars as an example of a cluster in the same region of the sky where the distance is known reliably from other information, as a test case to check the validity of the DR1 results, in particular for luminous stars. The distance we derive from DR1 is roughly correct, and the errors are similar to our LBV targets discussed in this paper.
} 

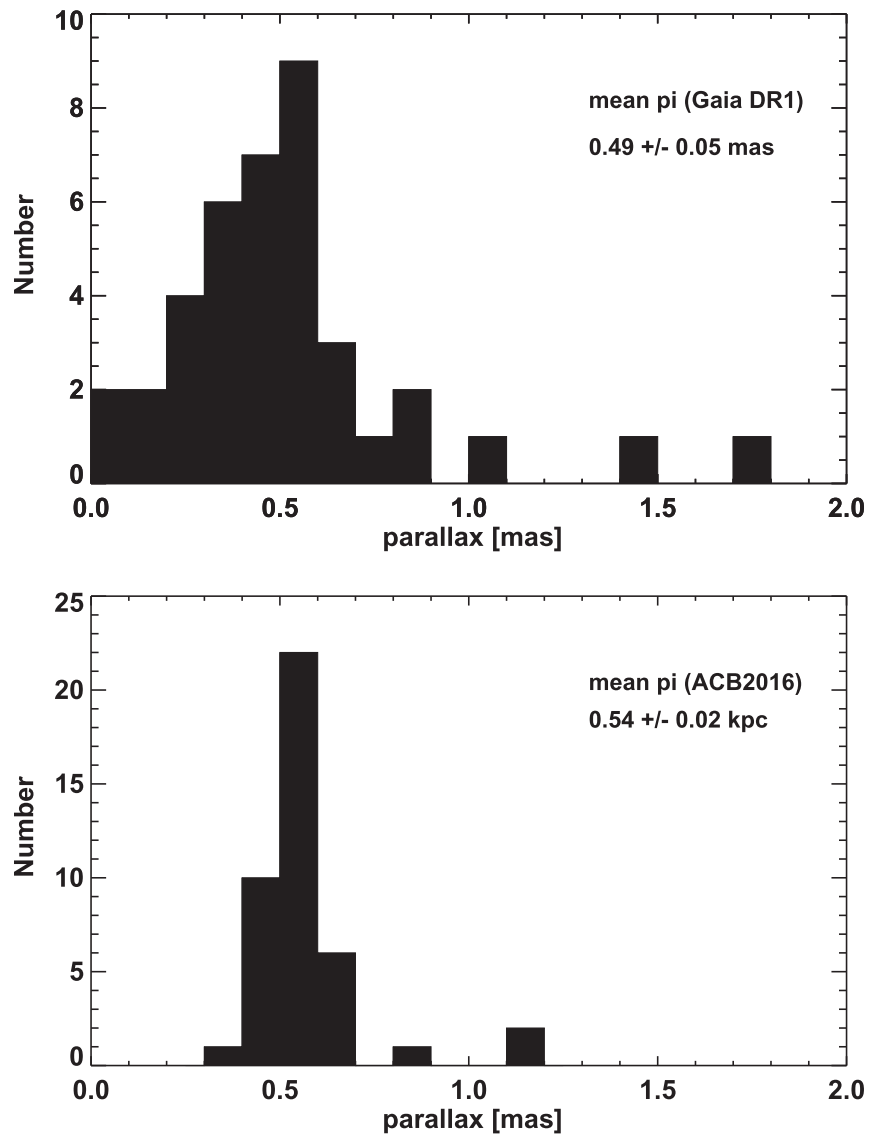

Figure 1. (Top) Distribution of Gaia DR1 parallaxes for O stars in the Carina star-forming region from Smith (2006a), all expected to be at $\pi \sim 0.45$ mas $(d \sim 2.2 \mathrm{kpc}$ ). (Bottom) Parallaxes according to a Milky Way prior-based calculation (Astraatmadja \& Bailer-Jones 2016).

median Gaia parallax error for these stars is $63 \%$, comparable to that for the four LBVs in our study sample.

Thus, the Gaia distances for the Carina O stars via a simple $1 / \pi$ estimator are consistent with expectation; the distances from the Milky Way prior-based method (ABJ) are perhaps slightly underestimated. In any event, this check provides a measure of validation that the Gaia distances for our target LBVs - also luminous stars at expected distances $\sim 2 \mathrm{kpc}$ or greater-should be reliable (see also Casertano et al. 2016; Jao et al. 2016; Stassun \& Torres 2016b; Davies et al. 2017). If so, the implications are important, as discussed next.

\section{Discussion}

Figure 2 shows LBVs on the HR Diagram, adapted from previous studies as noted in the caption. The red points show the luminosities for AG Car, HR Car, HD 168607, and Hen 3519 using previously adopted distances in the literature, whereas the blue points show how the luminosities are lowered if the nearer Gaia DR1 distances are adopted. For Hen 3-519, widely different luminosities are given for the same $8 \mathrm{kpc}$ distance by Davidson et al. (1993) and Smith et al. (1994) based on different assumed values of $E(B-V)$. We adopt an average of the two here, although this difference is small compared to the factor of 16 reduction in luminosity that results from the nearer distance.

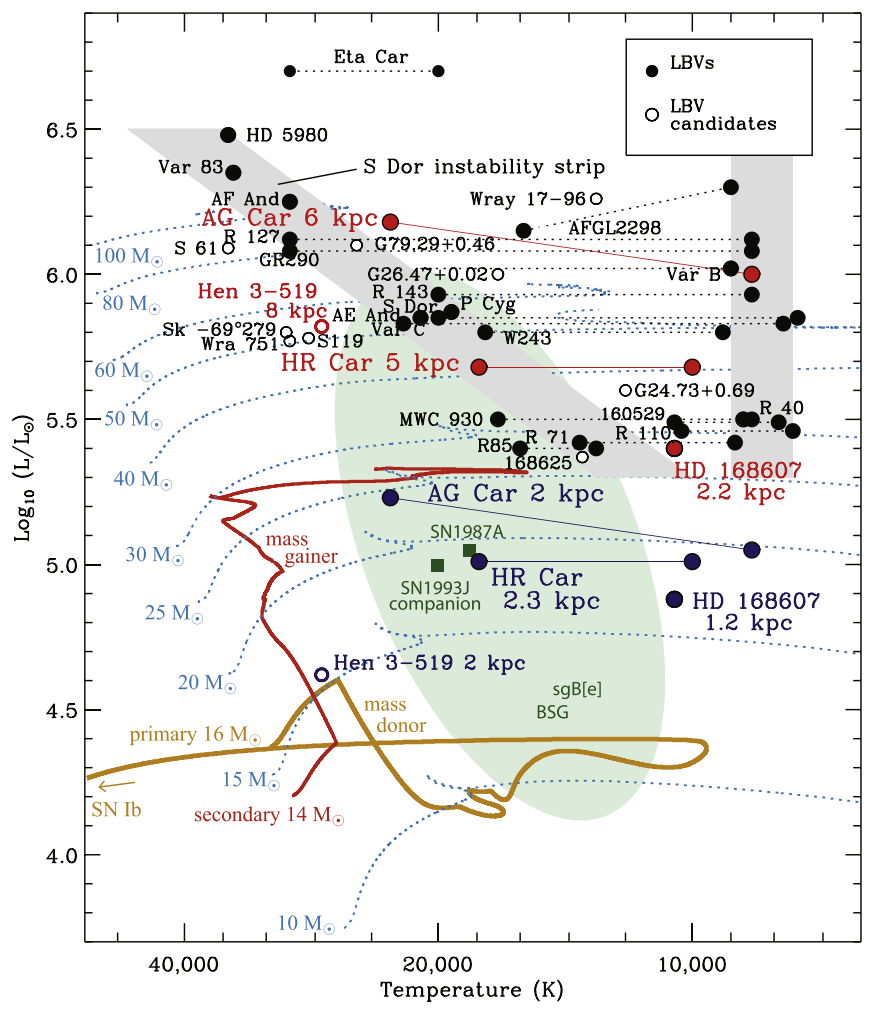

Figure 2. HR Diagram of LBVs. This is the same as in Smith \& Tombleson (2015), except that we have modified the entries for AG Car, HR Car, HD 168607, and Hen 3-519 as discussed in this paper. For all three, values from the literature with conventional distances are shown in red, and these are reduced according to the lower distances indicated by the new Gaia parallax measurements (shown in blue). For AG Car, we use the values derived from CMFGEN models assuming $d=6 \mathrm{kpc}$ from Groh et al. $(2009 \mathrm{~b}, 2011)$ in red, and the same temperatures but at $d=2 \mathrm{kpc}$ in blue. For HR Car we use values derived from CMFGEN models by Groh et al. (2009a) at $5 \mathrm{kpc}$ (red) and $2.3 \mathrm{kpc}$ (blue). For Hen 3-519 we use the temperature derived from CMFGEN models by Smith et al. (1994). We adopt the average of the two luminosities from Smith et al. (1994) and Davidson et al. (1993) at $8 \mathrm{kpc}$ (red), and at $2 \mathrm{kpc}$ (blue). For HD 168607, we use the values from Leitherer \& Wolf (1984). As in the original figure of Smith \& Tombleson (2015), single-star evolutionary tracks are from Brott et al. (2011), and example binary tracks are from Langer \& Kudritzki (2014).

As noted above, the nearer distance for HR Car is not in such severe disagreement with previous values, and for HD 168507, the distance agrees within the quoted uncertainty with previous estimates. It is interesting, though, that at the nearer $2.3 \mathrm{kpc}$ distance, the hotter state of HR Car is almost coincident on the HR Diagram with the progenitor of SN 1987A. Speculative comparisons between SN 1987A's progenitor and LBVs have been discussed before (Smith 2007). Future Gaia data releases will provide higher precision in the parallax and proper motion, so we postpone a more detailed discussion of HR Car and HD 168607 until then. For now, we discuss the basic implications of the substantially smaller distances for AG Car and Hen 3-519, where the Gaia distances - even with the relatively large uncertainty of DR1appear to be inconsistent with previous estimates, and where the possible implications for our understanding of LBVs are more severe. We acknowledge that the following discussion may need to be revisited if the improved Gaia DR2 measurements show the adopted DR1 values and uncertainties to be substantially incorrect. 


\subsection{AG Car as a Prototypical S Doradus Variable}

The new distance of $\sim 2 \mathrm{kpc}$ implied by AG Car's parallax is over three times closer than the usually assumed value. Humphreys et al. (1989) preferred $6.4-6.9 \mathrm{kpc}$, but at that distance, the parallax would be only 0.16 mas or less. Most authors have generally adopted a value of $6 \mathrm{kpc}$, so the $\sim 3$ times closer Gaia distance makes AG Car about 9 times less luminous than previously assumed. If true, this would have a tremendous impact on our interpretation of this star and possibly LBVs in general, and we explore these implications below. To place AG Car on the HR Diagram, we assume that the temperatures derived from CMFGEN models by Groh et al. (2009b, 2011) are the same, but that the bolometric luminosity scales by the square of the assumed distance. There are several immediate implications:

1. If AG Car's luminosity is lower by a factor of 9, it cannot be such a massive star. Previously, it sat along an evolutionary track for an initial mass of around $90-100 M_{\odot}$, but the lower luminosity corresponds to a single star of only $\sim 25 M_{\odot}$ initial mass. Single stars of this mass are not expected to approach the classical Eddington limit during their evolution. This raises profound questions about the source of AG Car's instability and mass loss, and the nature of the LBV instability itself.

2. Lowering its luminosity moves AG Car far off the $S$ Dor instability strip, which has been seen as the defining locus of LBVs in their hot state (Humphreys \& Davidson 1994). Yet, AG Car is an S Dor variable; in fact, it is a defining member of the class (Wolf \& Stahl 1982; Stahl 1986; Stahl et al. 2001). Since AG Car is considered a prototypical example of a classical high-luminosity LBV (Wolf \& Stahl 1982; Stahl 1986; Humphreys \& Davidson 1994), this raises important questions about our fundamental picture of LBVs, and suggests that the $\mathrm{S}$ Dor instability strip may not be as clean as previously thought — at least in the Milky Way. Stars far away from the S Dor instability strip may evidently be prone to LBV-like behavior as well, if the nearer Gaia distance is correct.

3 . The new luminosity is only $\log \left(L / L_{\odot}\right)=5.25$, implying an initial mass (if single) of only $\sim 25 M_{\odot}$, as noted above. This is now low enough that AG Car could have gone through a previous RSG phase. Several previous studies have concluded that dust in the shell nebula around AG Car resembles dust seen around massive RSGs (Smith et al. 1997; Voors et al. 2000). The possibility of a previous RSG phase has generally been discounted, since there are no RSGs observed at very high luminosities corresponding to initial masses above 35-40 $M_{\odot}$, whereas AG Car was thought to far exceed this limit. The new lower distance and $L$ removes this restriction. With strong mass loss during the RSG phase, even single-star models can produce LBV-like stars in this lower 20-25 $M_{\odot}$ initial mass range (Groh et al. 2013).

4. The lower distance also results in a lower mass for the nebula. AG Car's shell nebula was thought to have a huge total mass of 20-25 $M_{\odot}$, derived from the measured dust mass of $\sim 0.2-0.25 M_{\odot} \quad$ (Voors et al. 2000; Vamvatira-Nakou et al. 2015), an assumed gas-to-dust mass ratio, and a distance of $6 \mathrm{kpc}$. Since the dust mass depends linearly on the IR luminosity, the three times closer distance implies a nine times lower nebular mass of roughly $2.5 M_{\odot}$. The time averaged mass-loss rates needed to produce this nebula are therefore not as extreme as previously thought.

5. A closer distance for AG Car also has important consequences concerning the recently discussed environments of LBVs. Smith \& Tombleson (2015) pointed out that in general, LBVs seem strangely isolated from other young massive stars, and questioned their traditional role in evolution as very massive single stars in transition from O-type stars to WR stars. AG Car was surprisingly isolated for a star of 90-100 $M_{\odot}$ initial mass, being at least $30 \mathrm{pc}$ in projection from any other O-type star (Smith \& Tombleson 2015). Most of these neighboring O-type stars were thought to be in the foreground at around $2 \mathrm{kpc}$, while Chentsov \& Gorda (2004) and Hoekzema et al. (1992) were unable to identify any host cluster or association at $\sim 6 \mathrm{kpc}$ that might trace AG Car's birth environment. Moving AG Car to a distance of $2 \mathrm{kpc}$ in the Car OB association would mean that the projected separations on the sky to neighboring O-type stars are relevant. The large 30 pc separation would still be problematic if AG Car was a $100 M_{\odot}$ star-but it is far less problematic if AG Car has a much lower luminosity (and thus a lower initial mass and longer lifetime) than previously thought. ${ }^{5}$

So, with a nearer distance and lower luminosity, how can we understand AG Car's instability and mass loss, which have been used to help define the LBV class? In the traditional picture, the combination of a very high luminosity near the classical Eddington limit and rapid rotation bring the star to the so-called " $\Gamma \Omega$-Limit" during its post-main-sequence evolution (see Groh et al. 2011 and references therein). Indeed, AG Car is thought to be a rapid rotator based on evidence for a bipolar wind from spectropolarimetry, line profile shapes, and other diagnostics of rotation (Leitherer et al. 1994; Schulte-Ladbeck et al. 1994; Groh et al. 2006). However, this picture must be modified if AG Car is really as close as Gaia suggests. Stars of much lower luminosity and an initial mass around $\sim 25 M_{\odot}$ might conceivably develop a high $L / M$ ratio if they shed a large amount of mass in a previous RSG phase, so that similar ideas about envelope instability might still apply-but it is hard to imagine that a single star could shed all that mass while maintaining rapid rotation. Instead, it seems as though binary interaction must play some role in explaining AG Car, if it is less massive than previously thought. An example track of a mass-gainer on the HR diagram (Figure 2; evolutionary track from Langer \& Kudritzki 2014) would seem to explain the current properties of AG Car quite naturally. A stellar merger might produce an equally satisfying explanation (Podsiadlowski 2010). Such a star gains angular momentum from the mass it accretes from a companion or from a merger, rather than shedding all of its angular momentum in the RSG wind.

An important outstanding question is whether or not this rapid rotation gained from binary interaction plays some critical role in triggering the envelope instability that leads to $\mathrm{S}$ Doradus-like variability. Graefener et al. (2012) have hypothesized that rotation might be a trigger for the radius inflation of LBVs in their S Doradus cycle, if this is brought on by the density inversion below the photosphere that results from the Fe-opacity bump. Based on their surprisingly isolated environments (implying long lifetimes), Smith \& Tombleson (2015) suggested that most LBVs may be products of mass accretion or mergers in interacting binaries. This could potentially explain their anomalously high luminosities via rejuvenation,

\footnotetext{
5 One of the most pressing mysteries of LBVs is their relative isolation as compared to other massive stars (Smith \& Tombleson 2015). This argument was based largely on the cumulative distribution of separations between stars in the LMC, so this is not affected by the smaller distance and luminosity of AG Car.
} 
as well as rapid rotation late in life. If binary interaction is a key ingredient for the LBV instability, this is not necessarily limited to only the most massive and luminous stars.

Overall, the possibility of moving AG Car down on the HR diagram raises intriguing new questions about the blue supergiants (BSGs) and $\mathrm{B}[\mathrm{e}]$ supergiants corresponding to initial masses of $15-40 M_{\odot}$ (the green oval in Figure 2 ). This region includes persistently mysterious objects like the progenitor of SN 1987A, among others. Finding bona fide LBVs like AG Car and HR Car in this same region may focus our attention and generate new ideas about their significance in stellar evolution.

\subsection{Hen 3-519 at Low Luminosity}

Hen 3-519 has been studied far less intensively than AG Car. Less is known about it, and it is not a critical defining LBV like AG Car. The luminosity at $8 \mathrm{kpc}$ that we used in Figure 2 is the average of two values in the literature that disagree significantly (Davidson et al. 1993; Smith et al. 1994). We used the average of these to scale to the nearer distance of $\sim 2 \mathrm{kpc}$ indicated by the Gaia parallax. Renewed efforts to determine the fundamental physical parameters of this star are encouraged. The uncertainty in the measured parallax ( $\sigma_{\pi}$ in Table 1$)$ is larger than for the other stars, so the precise value of this new distance should be viewed with some degree of caution.

It is admittedly quite surprising that a star of such low luminosity would exhibit a peculiar emission-line spectrum that is so similar to the spectra of much more luminous stars, such as the Ofpe/WN9 stars in the LMC (Crowther et al. 1995). Aside from the lack of any known $\mathrm{S}$ Dor-like variability in Hen 3-519, many of the other comments above about AG Car may apply here as well-especially regarding the role of a previous RSG phase in forming its shell nebula, and the likely importance of binary evolution in explaining its current location on the HR diagram. Perhaps Hen 3-519 is not related to the LBVs at all. Its low luminosity might be more consistent with post-RSG evolution of a $10-15 M_{\odot}$ star, especially if it has encountered binary interaction (i.e., post common-envelope or post-RLOF). Regarding its LBV-like spectrum, it may be relevant to point out that even some evolved intermediate-mass (5-8 $M_{\odot}$ ) stars inside bipolar planetary nebulae have spectra that very closely resemble the unusual spectrum of the supermassive LBV $\eta$ Carinae (Balick 1989; Smith 2003). Evolved stars of vastly different luminosity and mass may achieve similar temperatures, densities, and composition in their winds.

\section{Summary and Conclusions}

We report the parallax distances and proper motions of the Galactic LBV stars AG Car, HR Car, and HD 168607, and the LBV candidate Hen 3-519, resulting from the Gaia DR1 first data release. These are the only Galactic LBVs included in DR1. The distances to all four objects are closer than traditionally assumed in the literature, suggesting lower intrinsic luminosities.

1. For HD 168607, the reduction in distance is about a factor of 2 , but the new distance is consistent with previous values within the uncertainty. We therefore postpone an evaluation of its properties until the higher precision that will be available later from Gaia.
2. For HR Car, the implied distance of $\sim 2.3 \mathrm{kpc}$ is more than a factor of 2 lower than the traditional value of $5 \pm 1 \mathrm{kpc}$, but the upper limit to the distance of $4.6 \mathrm{kpc}$ is still marginally consistent with the old value. It is interesting that at this closer distance, HR Car's position on the HR diagram is nearly identical to the progenitor of SN 1987A.

3. The prototypical, classical LBV star AG Carinae has a Gaia parallax indicating a distance of only $\sim 2 \mathrm{kpc}$ - three times closer than previously thought, making it nine times less luminous. The upper limit to its distance is inconsistent with the usually adopted value of 6.4-6.7 kpc (Humphreys et al. 1989). It now lies along a track of a $25 M_{\odot}$ star, rather than a 90-100 $M_{\odot}$ star. If correct, this has dramatic implications for the interpretation of this star and its nebula. In particular, AG Car may have gone through a previous RSG phase, and it is likely to be a product of binary interaction. Moreover, since AG Car is regarded as a defining member of the $\mathrm{S}$ Doradus class and a classical high-luminosity LBV, its lower luminosity and mass have profound consequences for our traditional view of LBVs in general.

4. For Hen 3-519, the distance implied by the Gaia parallax is also about $2 \mathrm{kpc}$, which is $\sim 4$ times closer than previously thought (making the star 16 times less luminous). This moves it far away from the traditional locus of LBVs on the HR diagram. Its origin and evolutionary state remain unclear, but its nebula may be the product of previous RSG mass loss or binary interaction.

Given that these stars were thought to be among the most luminous stars in the Milky Way and have shaped our views of LBVs for the last 30 years, it seems likely that Gaia distances for the remaining LBVs may instigate a re-evaluation of our standard view of LBVs. More precise values of the parallax for these four objects and for a larger number of Galactic LBVs will be available soon; the results reported here may hint at a coming upheaval in our understanding of massive-star evolution, even if they are regarded as preliminary.

An obvious avenue for further investigation is whether there are similar low-luminosity stars in the LMC or other nearby galaxies that have LBV-like spectra or variability, but that have evaded detection because they are faint. On the other hand, this does not undermine the properties of existing LBVs known in the LMC and SMC, since their distances are not so uncertain; the S Dor strip still seems to work for them. It will be interesting to investigate why there are stars that appear to be classical S Doradus-like LBVs in the Milky Way that are so far below the traditional S Dor instability strip. Perhaps metallicity plays a key role, or perhaps samples in the LMC are incomplete at lower luminosity.

We thank Megan Kiminki for double-checking the Gaia distances for O-type stars in the Carina Nebula and for helpful discussions. Support for N.S. was provided by the National Science Foundation (NSF) through grants AST-1210599 and AST-1312221 to the University of Arizona. K.G.S. acknowledges partial support from NSF PAARE grant AST-1358862. This work has made use of data from the European Space Agency (ESA) mission Gaia (http://www.cosmos.esa.int/ gaia), processed by the Gaia Data Processing and Analysis Consortium (DPAC, http://www.cosmos.esa.int/web/gaia/ dpac/consortium). Funding for the DPAC has been provided by national institutions, in particular the institutions participating in the Gaia Multilateral Agreement. 


\section{References}

Astraatmadja, T. L., \& Bailer-Jones, C. A. L. 2016, ApJ, 833, 119

Bailer-Jones, C. A. L. 2015, PASP, 127, 994

Balick, B. 1989, AJ, 97, 476

Boffin, H. M. J., Rivinius, T., Merand, A., et al. 2016, A\&A, 593, A90

Brott, I., Evans, C. J., Hunter, I., et al. 2011, A\&A, 530, A115

Gaia Collaboration, Brown, A. G. A., Vallenari, A., et al. 2016, A\&A, 595, A2

Casertano, S., Riess, A. G., Bucciarelli, B., \& Lattanzi, M. G. 2016, arXiv: 1609.05175

Chentsov, E. L., \& Gorda, E. S. 2004, AstL, 30, 461

Conti, P. S. 1984, IAUS, 105, 233

Crowther, P. A., Hillier, D. J., \& Smith, L. J. 1995, A\&A, 293, 172

Davidson, K. 1987, ApJ, 317, 760

Davidson, K., Humphreys, R. M., Hajian, A., \& Terzian, Y. 1993, ApJ, 411,336

Davies, G. R., Lund, M. N., Miglio, A., et al. 2017, arXiv:1701.02506

de Koter, A., Lamers, H. J. G. L. M., \& Schmutz, W. 1996, A\&A, 306, 501

Graefener, G., Owocki, S. P., \& Vink, J. S. 2012, A\&A, 538, A40

Groh, J. H., Damineli, A., Hillier, D. J., et al. 2009a, ApJL, 705, L25

Groh, J. H., Hillier, D. J., \& Damineli, A. 2006, ApJL, 638, L33

Groh, J. H., Hillier, D. J., Damineli, A., et al. 2009b, ApJ, 698, 1698

Groh, J. H., Hillier, D. J., \& Damineli, A. 2011, ApJ, 736, 46

Groh, J. H., Meynet, G., Georgy, C., \& Ekström, S. 2013, A\&A, 558, 131

Hoekzema, N. M., Lamers, H. J. G. L. M., \& van Genderen, A. M. 1992, A\&A, 257,118

Humphreys, R. M., \& Davidson, K. 1994, PASP, 106, 1025

Humphreys, R. M., Lamers, H. J. G. L. M., Hoekzema, N., \& Cassatella, A. 1989, A\&A, 218, L17

Jao, W.-C., Henry, T. J., Riedel, A. R., et al. 2016, ApJL, 832, L18

Langer, N., \& Kudritzki, R. P. 2014, A\&A, 564, A52

Leitherer, C., \& Wolf, B. 1984, A\&A, 132, 151

Leitherer, C., Allen, R., Altner, B., et al. 1994, ApJ, 428, 292

Lindegren, L., Lammers, U., Bastian, U., et al. 2016, A\&A, 595, A4

Mauerhan, J. C., Smith, N., Filippenko, A. V., et al. 2013, MNRAS, 430, 1801

Mauerhan, J. C., Van Dyk, S. D., Graham, M. L., et al. 2015, MNRAS, 447, 1922

Owocki, S. P., Gayley, K. G., \& Shaviv, N. J. 2004, ApJ, 616, 525

Owocki, S. P., \& Shaviv, N. J. 2016, MNRAS, 462, 345

Pasquali, A., Nota, A., Smith, L. J., et al. 2002, AJ, 124, 1625
Podsiadlowski, P. 2010, NewAR, 54, 39

Prieto, J. L., Rest, A., Bianco, F. B., et al. 2014, ApJ, 787, 8

Rest, A., Prieto, J. L., Walborn, N. R., et al. 2012, Natur, 482, 375

Robberto, M., \& Herbst, T. M. 1998, ApJ, 498, 400

Schulte-Ladbeck, R. E., Clayton, G. F., Hillier, D. J., Harries, T. J., \& Howarth, I. D. 1994, ApJ, 429, 846

Sesar, B., Fouesneau, M., Price-Whelan, A. M., et al. 2016, arXiv:1611.07035

Smith, L. J, Crowther, P. A., \& Prinja, R. K. 1994, A\&A, 281, 833

Smith, L. J, Stroud, M. P., Esteban, C., \& Vilchez, J. M. 1997, MNRAS, 290,265

Smith, N. 2003, MNRAS, 342, 383

Smith, N. 2006a, MNRAS, 367, 763

Smith, N. 2006b, MNRAS, 644, 1151

Smith, N. 2007, MNRAS, 133, 1034

Smith, N. 2008, Natur, 455, 201

Smith, N. 2013, MNRAS, 429, 2366

Smith, N. 2014, ARAA, 52, 487

Smith, N., \& Owocki, S. P. 2006, ApJL, 645, L45

Smith, N., \& Tombleson, R. 2015, MNRAS, 447, 602

Smith, N., Vink, J. S., \& de Koter, A. 2004, ApJ, 615, 475

Smith, N., Li, W., Silverman, J. M., Ganeshalingam, M., \& Filippenko, A. V. 2011, MNRAS, 415, 773

Stahl, O. 1986, A\&A, 164, 321

Stahl, O., Jankovics, J., Kovacs, J., et al. 2001, A\&A, 375, 54

Stahl, O., Wolf, B., Klare, G., et al. 1983, A\&A, 127, 49

Stassun, K. G., Collins, K. A., \& Gaudi, B. S. 2016, arXiv:1609.04389

Stassun, K. G., \& Torres, G. 2016a, AJ, 152, 180

Stassun, K. G., \& Torres, G. 2016b, ApJL, 831, L6

Sterken, C., Arentoft, T., Duerbeck, H. W., \& Brogt, E. 1999, A\&A, 349, 532

Vamvatira-Nakou, C., Hutsemekers, D., Royer, P., et al. 2015, A\&A, 578, A108

Van Dyk, S. D., \& Matheson, T. 2012, in Eta Carinae and the Supernova Impostors, ed. R. M. Humphreys \& K. Davidson (Berlin: Springer), 249

van Genderen, A. M., Robijn, F. H. A., van Esch, B. P. M., \& Lamers, H. J. G. 1991, A\&A, 246, 407

van Genderen, A. M., van den Bosch, F. C., Dessing, F., et al. 1992, A\&A 264, 88

Voors, R. H. M., Waters, L. B. F. M., Morris, P., et al. 2000, A\&A, 356, 501

Wolf, B. 1989, A\&A, 217, 87

Wolf, B., \& Stahl, O. 1982, A\&A, 112, 111 